\title{
Detection and analysis of heat focus associated with soil use in the Munim River watershed
}

\author{
Renato Oliveira da Silva ${ }^{l}$, Kamilla Andrade Oliveiral ${ }^{l}$, Telmo José Mendes ${ }^{l}$, \\ Carlos Augusto Cardoso Silval, Ana Karla da Silva Oliveiral, Washington da Silva Sousa ${ }^{l}$
}

\footnotetext{
${ }^{1}$ Universidade Federal do Maranhão, Maranhão, Brasil

*e-mail: oliveira.rennato@ hotmail.com; kamilla.andrade@ufma.br; telmo.mendes@ufma.br; carlosaugusto8435@gmail.com; karlinha_oliveira95@hotmail.com; wssousa@gmail.com
}

\begin{abstract}
Forest fires are mostly caused by anthropogenic actions and have a devastating potential in the environment. The Munim River Watershed has experienced strong economic growth linked to the exploitation of natural resources. This research aimed to identify hot flashes in the Munim River Watershed and associate them with land use. For land use analysis, scenes 220/62 and 220/63 were used. Through the use of ArcMap 10.5 software, different digital image processing practices were carried out, which can be described in three main steps: Preprocessing, Highlighting and classification, where the defined classes were: dense vegetation, thin vegetation, exposed soil, clouds, and, water bodies. The data obtained for the analysis of heat sources in the Munim River Watershed region were obtained on the electronic platform of the National Institute for Space Research (INPE) on the period from 2008 to 2018 based on data of satellites by NOAA, GOES, AQUA, NPP METOP, MSG, and TERRA series. Among the years analyzed, 2011 was the year with the lowest number of hot spots, a total of 2,114, whereas 2015 stood out with the highest number of hot spots, totaling 14,048. By combining the hot spots with land use in the year 2015, it was evidenced that most of them were identified in areas classified as thin vegetation with $41.95 \%$ and in areas of exposed soil with $39.76 \%$ and in smaller amounts in areas of dense vegetation with $16.99 \%$ of allocated points. The cities of Chapadinha, Afonso Cunha, Coelho Neto, Buriti, São Benedito do Rio Preto and Urbano Santos were the ones that indicated the largest number of hot spots, being classified as Very High, High and Medium intensity. In the years 2009 and 2011 the lowest focus intensities were identified in the Munim River Watershed, being quantified at 2,690 and 2,114, respectively.
\end{abstract}

Keywords: use of the soil, natural resources, heat spots 